\title{
Epidemiology of Nontuberculous Mycobacteriosis
}

\author{
Jennifer Adjemian, $\mathrm{PhD}^{1,2}$ Shelby Daniel-Wayman, BS ${ }^{1}$ Emily Ricotta, ScM ${ }^{1}$ \\ D. Rebecca Prevots, PhD, MPH ${ }^{1}$
}
${ }^{1}$ Epidemiology Unit, Laboratory of Clinical Immunology and Microbiology, Division of Intramural Research, National Institute of Allergy and Infectious Diseases, National Institutes of Health, Bethesda, Maryland
${ }^{2}$ Commissioned Corps, United States Public Health Service, Rockville, Maryland

\begin{abstract}
Address for correspondence Jennifer Adjemian, PhD, Epidemiology Unit, Laboratory of Clinical Immunology and Microbiology, Division of Intramural Research, National Institute of Allergy and Infectious Diseases, National Institutes of Health, Qrts 15 B-1, 8 West Drive, MSC 2665, Bethesda, MD 20892-2665 (e-mail: Jennifer.adjemian@nih.gov).
\end{abstract}

Semin Respir Crit Care Med 2018;39:325-335.

\begin{abstract}
Keywords

- epidemiology

- nontuberculous mycobacteria

- pulmonary disease
\end{abstract}

Nontuberculous mycobacteria (NTM) are ubiquitous environmental bacteria commonly found in water and soil and can cause pulmonary and extrapulmonary infections in susceptible individuals. NTM are increasingly recognized as important causes of human morbidity and mortality, with several recent studies highlighting significant increases in prevalence of pulmonary NTM (PNTM) disease in the United States and globally. ${ }^{1-6}$ While more than 180 mycobacterial species have been identified to date (full list can be found at http://www.bacterio.net/mycobacterium.html), significant epidemiologic variations exist by species, including differences in geographic distribution and risk factors. ${ }^{7-9}$ Here, we review the epidemiology of PNTM disease in the United States, including differences by mycobacterial species and known host and environmental factors that appear to impact disease risk.

\section{Prevalence in the United States}

Understanding the epidemiologic patterns and trends of PNTM in the United States is an ongoing challenge because PNTM is a reportable condition in only 11 states. Unlike Mycobacterium tuberculosis, which causes tuberculosis (TB), pulmonary infection due to NTM is not a nationally notifiable disease in most of the United States, impeding the systematic monitoring of its incidence, prevalence, and temporal trends. Instead, available U.S. estimates for PNTM prevalence are generated through studies utilizing other data sources, including local surveillance efforts, single-site studies, clinical surveys, disease registries, and large laboratory and claims-based datasets. Additionally, the case definition for PNTM disease established by the American Thoracic Society (ATS)/Infectious Disease Society of America (IDSA) ${ }^{10}$ requires strict microbiological, 10.1055/s-0038-1651491. ISSN 1069-3424. 
radiological, and clinical criteria to be met, in an effort to rule out transient infection. However, these stringent requirements present further challenges in identifying all PNTM disease cases and thus estimating its actual prevalence, as access to and use of the medical care and services needed to meet these criteria vary greatly across populations and especially by insurance coverage, race/ethnicity, and socioeconomic status. ${ }^{11}$

To address these challenges, numerous studies utilizing each of the data source types listed above-each with its own advantages and limitations-along with various PNTM case definitions ranging from single isolation studies to those meeting full ATS/IDSA criteria, have been conducted to address questions regarding the epidemiology and prevalence of PNTM in the United States.

\section{PNTM Prevalence in the General Population}

Because PNTM is a relatively rare condition, large population-based datasets from various healthcare systems have been particularly important in describing the burden of NTM (-Table 1). The first nationwide estimates of PNTM prevalence were generated using a population-based sample of nearly 3 million U.S. Medicare beneficiaries aged 65 and older from 1997 to $2007^{1}$ (-Table 1). This study found that prevalence of PNTM, as estimated from the presence of at least one International Classification of Disease (ICD)-9 code specific for PNTM, increased significantly across all regions of the United States by $8.2 \%$ per year, from 20 to 47 cases per 100,000 persons. This estimate is similar to what was reported in a Canadian study that identified an annual increase in the isolation prevalence of PNTM of $8.4 \%$ per year from 1997 to 2003. ${ }^{3}$ Because PNTM is a chronic condition, and persons may have PNTM in more than 1 year, the overall period prevalence of PNTM was estimated at 112 cases per 100,000 persons across all states. However, significant geographic variations in prevalence were identified by state and race/ethnicity. The highest period prevalence was in the state of Hawaii at 396 cases per 100,000 persons, followed by California, Florida, and Louisiana which had a prevalence that was approximately twofold greater than the national average (-Fig. 1). In addition, while more than $90 \%$ of the PNTM cases identified were white, persons identified as Asians/Pacific Islanders were at significantly greater risk for disease, with a prevalence of twofold that of whites. Limitations of this study included a lack of mycobacterial laboratory data and the reliance on ICD-9 codes to identify patients with PNTM disease, which has been shown to miss $\sim 25$ to $75 \%$ of cases. ${ }^{12,13}$ While this study may therefore underestimate the true prevalence of PNTM disease, the patterns and trends identified here provided significant insight into its epidemiology in the United States.

Two additional population-based studies were conducted in large health maintenance organizations (HMO), representing a total of five sites in the United States: one at four HMOs in different areas of the country, including parts of Southern California, Colorado, Seattle, and Pennsylvania, ${ }^{13}$ and a second study in Hawaii, which has a high prevalence of NTM as well as a high proportion of the population with Asian/Pacific Islander ancestry $^{2}$ (-Table $\mathbf{1}$ ). While these regional studies repre- sented fewer people and geographic areas than the Medicare study, the inclusion of mycobacteriology laboratory data and other clinical and demographic features across all age groups resulted in robust prevalence estimates. These studies both found that Mycobacterium avium complex (MAC) comprised $\sim 80 \%$ of all cases and that PNTM was more common in older adults, with an increased prevalence among women relative to men (1.1- to 1.9-fold higher). Both studies also found a 2- to 2.9-fold higher prevalence of NTM compared with TB during the same time period. ${ }^{2,13}$ In the study of four health care systems, annual prevalence among all ages ranged from 1.4 to 6.6 per 100,000 persons across the four regions and increased from 1994 to 2006 by $2.6 \%$ per year among men and $2.9 \%$ per year among women. ${ }^{13}$

The Hawaii HMO study represented more than 300,000 beneficiaries enrolled in Kaiser Permanente Hawaii (KPH) from 2005 through 2013 and estimated prevalence and trends by species and race/ethnicity. ${ }^{2}$ Here, the annual prevalence of PNTM isolation across all ages showed a relative increase of $6 \%$ per year, from 9 to 19 cases per 100,000 persons over the study period; when evaluated by species, the increase was found only for MAC-associated PNTM. The overall period prevalence was 122 cases per 100,000 persons for this time period and increased to 696 cases per 100,000 persons among those aged 65 years and older-double of that was estimated for Hawaii using insurance claims codes in the U.S. Medicare study. ${ }^{1}$ of greatest interest in the $\mathrm{KPH}$ study $^{2}$ was differences by race/ ethnicity, with the highest period prevalence observed among persons identified as Japanese, Korean, Chinese, or Vietnamese, with a prevalence of 293 to 336 cases per 100,000 , compared with a prevalence of only 156 cases per 100,000 persons for persons identified as white. Persons who identified as Native Hawaiians or Pacific Islanders had the lowest PNTM prevalence at 50 cases per 100,000 persons. Gender differences in NTM prevalence were also noted by racial/ethnic group. Among persons who identified as Vietnamese, NTM was more prevalent in men than in women (568 vs. 105 cases per 100,000 persons), while among those who identified as Japanese, NTM was more prevalent in women than in men (378 vs. 287 cases per 100,000 persons); in all other racial/ethnic groups, prevalence did not differ greatly by sex. The NTM isolation prevalence was consistently double the prevalence of PNTM cases meeting the definition for disease (i.e., two or more positive mycobacterial cultures), regardless of age, sex, or race/ethnicity. Differences in prevalence by NTM species were also noted by race/ethnicity, with Japanese and Filipinos twice as likely to have $M$. abscessus isolated and Vietnamese more likely to have MAC, M. abscessus, and M. fortuitum group isolated relative to other racial/ethnic groups. ${ }^{2}$

Population-based estimates of PNTM were also derived for three counties in North Carolina, from which researchers obtained clinical and demographic data for all samples tested from 2006 to 2010 ( - Table 1). In this study, the average annual prevalence was 9.4 per 100,000 persons, and was similar among men and women. ${ }^{14}$ However, significant differences were observed by sex and race, whereby among women, prevalence was highest among white women at 10.2 per 100,000 persons, while among men, prevalence was highest 


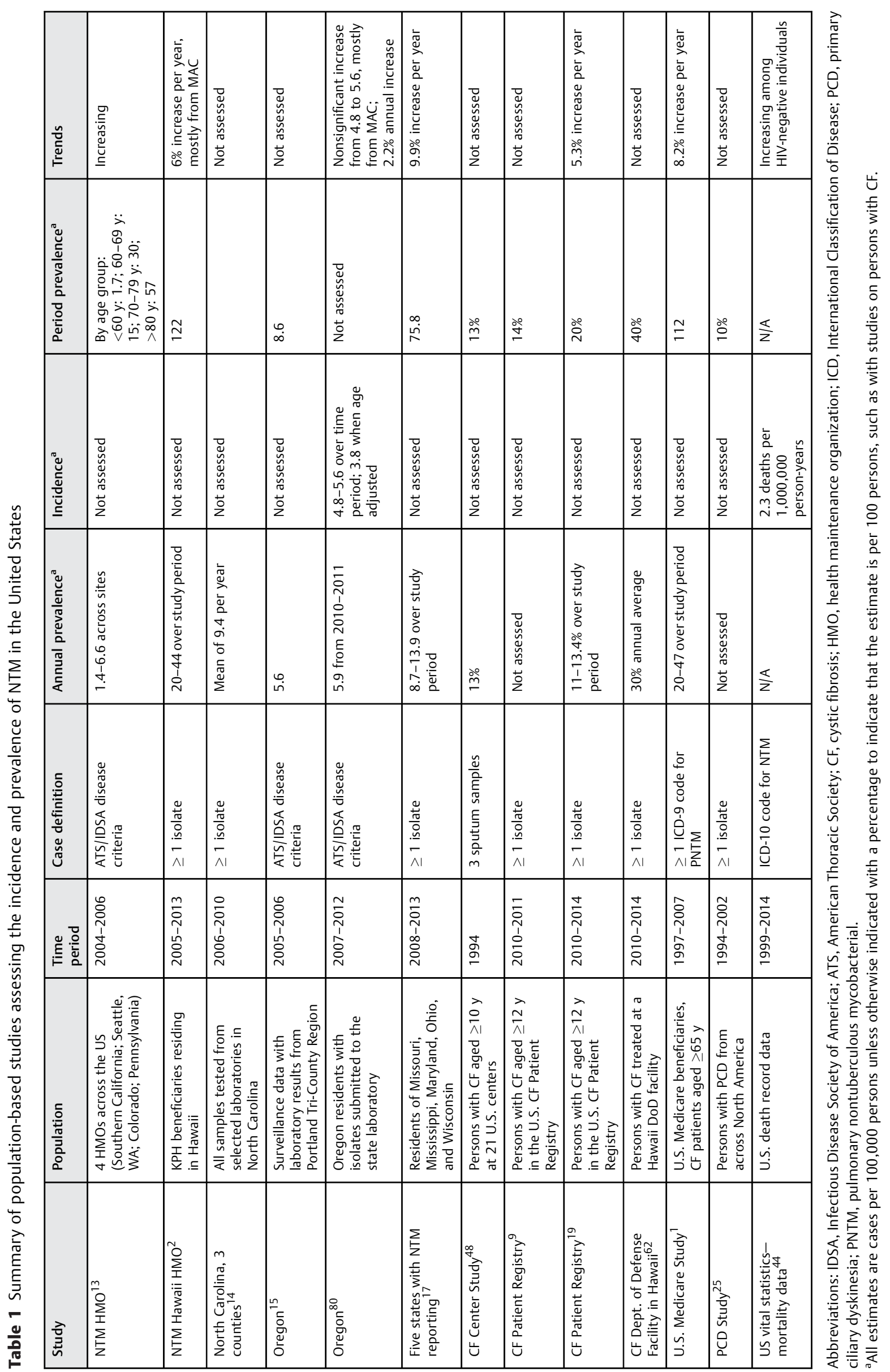




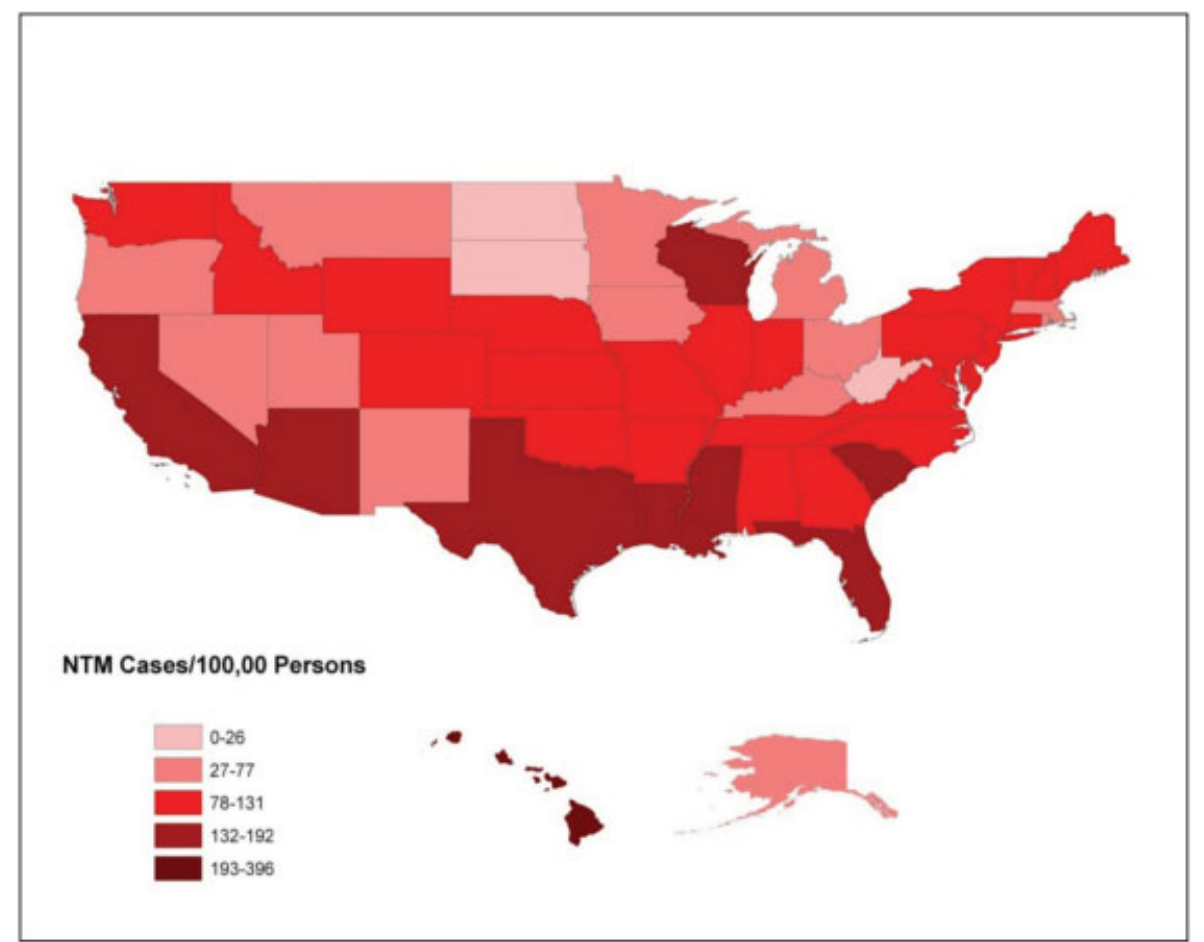

Fig. 1 Period prevalence of PNTM reported among U.S. Medicare beneficiaries aged 65 years and older by state, $1997-2007 .{ }^{1}$ PNTM, pulmonary nontuberculous mycobacterial.

for black men at $\sim 7$ per $100,000 .{ }^{14}$ Although estimates were not adjusted for age, observed rates by age showed a nearby twofold increased prevalence at nearly 40 per 100,000 persons among whites aged $>60$ years compared with nearly 20 per 100,000 persons among blacks in the same age group. ${ }^{14}$

In Oregon, a state-wide surveillance system was implemented and used to generate population-based PNTM estimates ( - Table 1), where near-complete identification of all NTM isolates was achieved by state-mandated reporting of NTM for a 2-year period from 2005 to $2006 .{ }^{15}$ This allowed for complete chart review with estimation of the prevalence of cases with microbiologic criteria meeting the ATS case definition for PNTM, as well as validating the specificity of these criteria for identifying those patients who met the full ATS NTM disease criteria among patients in the Portland TriCounty metropolitan region. Considering only those who met full ATS disease criteria, the prevalence of NTM disease in this region was found to be 8.6 cases per 100,000 persons over the 2-year period; however, this is an underestimate, as $30 \%$ of patients lacked an evaluable record. Of those meeting microbiologic criteria, $87 \%$ also fulfilled the full case definition, $88 \%$ of who had disease caused by MAC. ${ }^{10,15}$

In a follow-up study in Oregon, data were also collected from all microbiologic laboratories serving the state from 2007 to $2012^{16}$ ( - Table 1 ). Using the previous data from 2005 to 2006 to exclude prevalent cases, ${ }^{15}$ they found annual incidence rates that ranged from 4.8 per 100,000 persons in 2007 to 5.6 per 100,000 persons in $2012 .^{16}$ Incidence was higher among women overall, but among persons aged $<60$ years, incidence was higher among men compared with women. ${ }^{16}$ The close correspondence of the incidence rates with the prevalence estimated from this and other studies suggests that, because most patients only have a single pulmonary specimen tested for NTM, the observed prevalence also reflects incidence, or the first diagnosis of disease.

While PNTM disease is not a nationally reportable condition in the United States, several states do have NTM infection or disease listed as a notifiable condition. In 2015, electronic data from five of these states with reporting requirements were used to estimate trends in NTM infection, defined as at least one isolate of NTM (including both pulmonary and extrapulmonary sources; - Table 1). For all states combined, the average annual age-adjusted prevalence increased from 8.7 to 13.9 cases per 100,000 persons between 2008 and $2013 .^{17}$ The increase was most marked in the $>50$ age group, with an annual percent change of $11.8 \%{ }^{17}$

Recently, to estimate the overall burden of PNTM across all age groups, in the United States, investigators used prevalence estimates from Medicare data and clinician/patient survey data to generate a national prevalence estimate across all age groups weighted by the age distribution of the U.S. population. ${ }^{18}$ Additionally, by combining these data with data on treatment cost by type of insurance, this study estimated a total of 86,244 NTM cases in the United States in 2010, at a cost of $\$ 815$ million. Case numbers were highest among coastal and southeastern states, with California, Florida, Texas, New York, and Pennsylvania (which ranged from an estimated 3,969 to 12,544 cases in 2010) comprising $44 \%$ of the annual costs in the United States. When the previously estimated annual increase in PNTM prevalence of $8.2 \%{ }^{1}$ was applied, projected national case numbers for 2014 increased to 181,037, with an associated annual cost of $\$ 1.7$ billion. $^{18}$ 


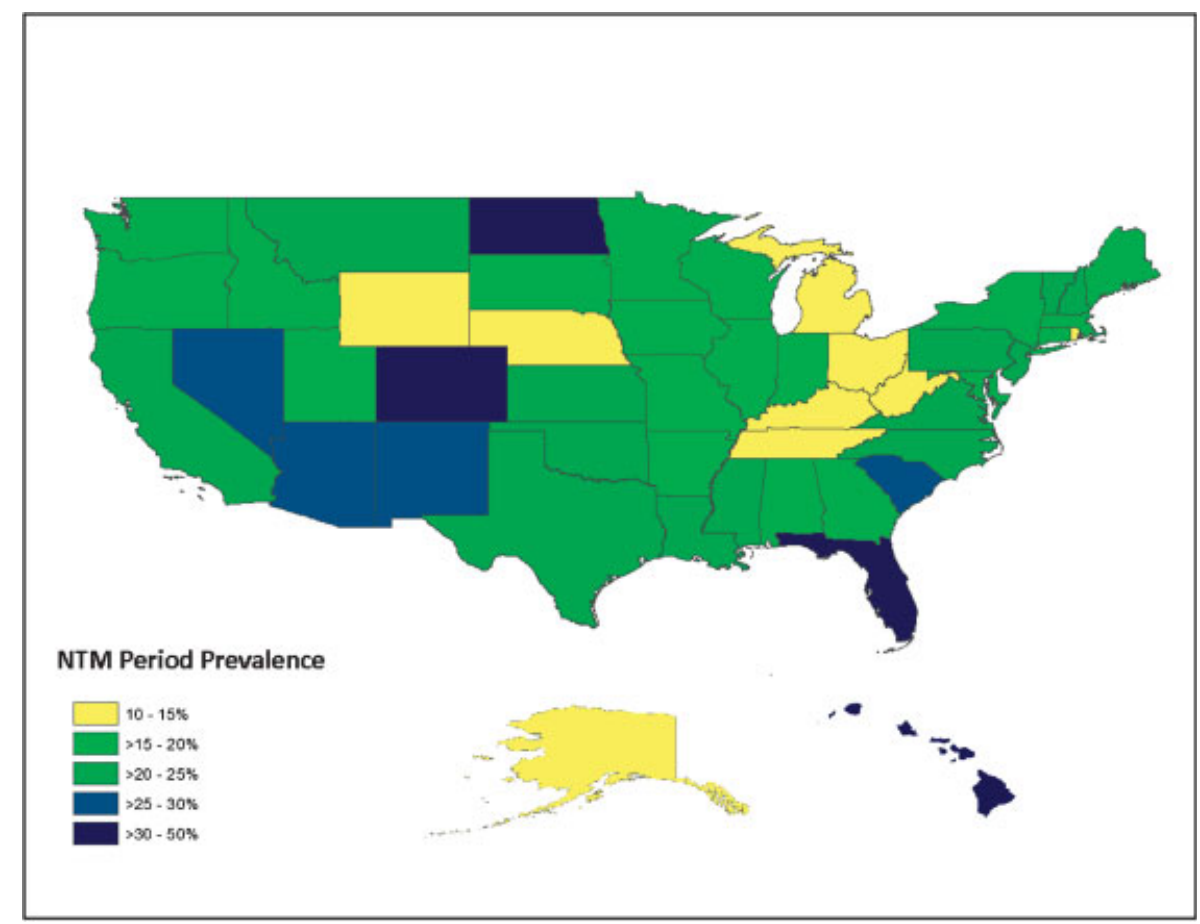

Fig. 2 Prevalence of PNTM isolated from persons with cystic fibrosis in the United States, 2010-2014. ${ }^{19}$ PNTM, pulmonary nontuberculous mycobacterial.

\section{PNTM Prevalence in High-Risk Populations}

Although PNTM remains rare in the general population, individuals with certain comorbid conditions and/or underlying pulmonary genetic defects are at significantly greater risk of acquiring PNTM. Among persons with cystic fibrosis (CF), who are highly susceptible to opportunistic pathogens, prevalence averages $12 \%$ nationally, and is up to $50 \%$ in some geographic regions ${ }^{9,19}$ (- Fig. 2). Studies indicate that like in the general population, PNTM prevalence in the CF population has been identified and/or reported with greater frequency over time, from just $1 \%$ in a limited study in $1984^{20}$ up to $14 \%$ across a 2-year period from 2010 to 2011 among all persons with CF in the United States. ${ }^{9}$ This latter study utilized data from the CF Foundation's national patient registry (CFPR), which since 2010 has collected detailed mycobacterial laboratory data on $>90 \%$ of all persons with $\mathrm{CF}$ in the United States, facilitating longitudinal epidemiologic analyses of $\mathrm{NTM}^{9,19,21}$ (- Table 1). Among those with PNTM isolated, 61\% had MAC and 39\% had M. abscessus, a species distribution that has remained stable over time, but varies greatly by state (-Fig. 3). ${ }^{9}$ More recently, an analysis conducted using CFPR data through 2014 demonstrated a

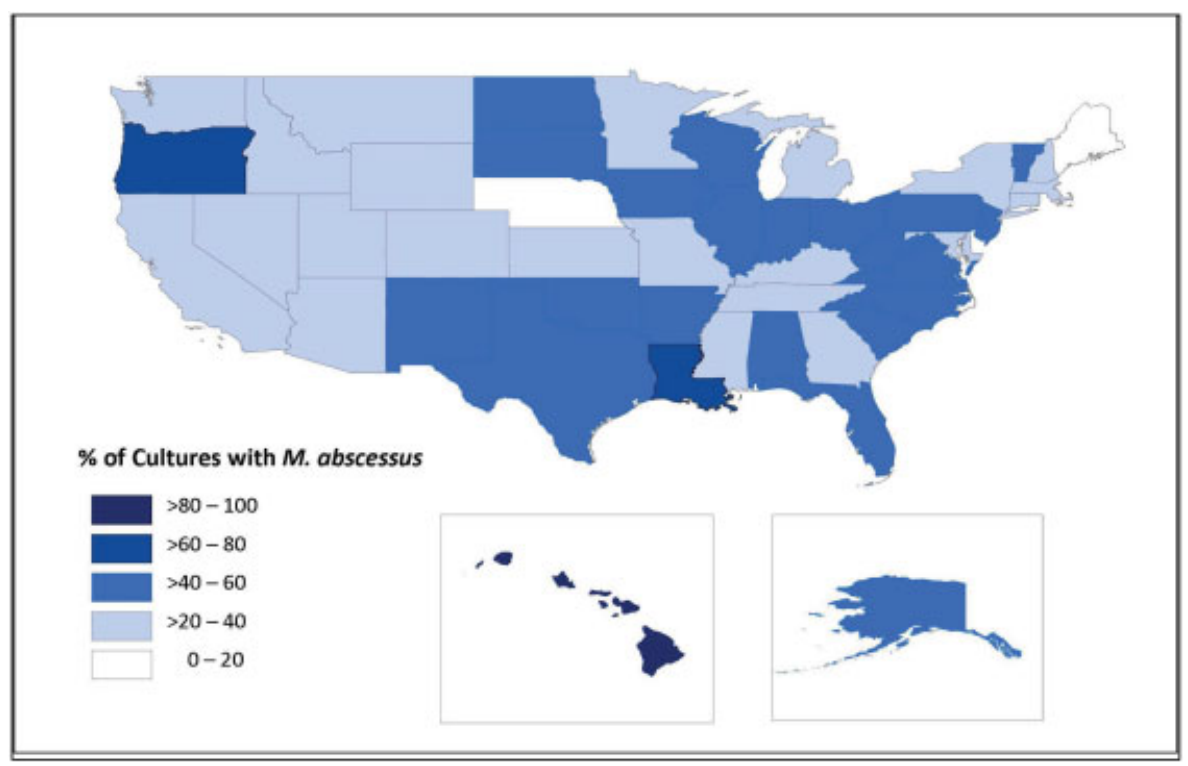

Fig. 3 Percentage of positive mycobacterial cultures attributed to M. abscessus among persons with cystic fibrosis in the United States, 20102011. ${ }^{9}$ PNTM, pulmonary nontuberculous mycobacterial. 
significant relative increase in PNTM isolation by $5 \%$ per year, from $11 \%$ in 2010 to over $13 \%$ in $2014^{19}$ (- Table 1 ). This study also showed that over a 5-year observational period, $20 \%$, or one in five persons with $\mathrm{CF}$, had a positive mycobacterial culture at least once. These individuals were more likely to receive their initial CF diagnosis after age 30 years, with a PNTM prevalence nearly double that of those diagnosed with CF by age 3. Additionally, those with M. abscessus were younger at initial CF diagnosis, and were more frequently homozygous for the p.Phe508del mutation, which typically reflects a more severe $\mathrm{CF}$ phenotype, ${ }^{22}$ relative to those with MAC-although not compared with CF study participants without PNTM isolated. ${ }^{19}$

Other populations that are highly susceptible to PNTM include persons with chronic comorbid conditions that affect the lungs such as chronic obstructive pulmonary disorder (COPD), bronchiectasis, and primary ciliary dyskinesia (PCD), or with conditions where treatment with immunosuppressants or immunomodulatory agents is common, such as rheumatoid arthritis (RA). In the PNTM study on U.S. Medicare beneficiaries, ${ }^{1}$ among women with PNTM, claims for RA and bronchiectasis were 1.5 and 1.7 times more common, respectively, while among men with PNTM, claims for diabetes and lung cancer were each 1.5 times more common than those without PNTM. In this same study, the prevalence of COPD, which included ICD-9 codes for COPD, emphysema, and chronic bronchitis, was $87 \%$ among PNTM cases and $44 \%$ among noncases; these estimates did not vary significantly by sex or geographic region. ${ }^{1}$ Similarly, in a retrospective cohort study conducted using U.S. hospital-based patient encounters from 176 hospitals nationwide, $28 \%$ of patients with NTM-positive cultures had COPD, and $11 \%$ had bronchiectasis. ${ }^{23}$ Another population-based study conducted among older adults in Ontario diagnosed with RA found that individuals with NTM disease were twice as likely to be using anti-tumor necrosis factor (anti-TNF) therapy for treatment of their RA than those without NTM disease. ${ }^{24}$ In a study evaluating persons with confirmed PCD, among those over age 30 years, 10\% had NTM isolated $^{25}$ ( - Table 1). Finally, in a recent study among persons living in Taiwan and with Sjögren's syndrome, a systemic chronic inflammatory disorder characterized by lymphocytic infiltrates in the exocrine glands, those treated with immunosuppressants were 25 times more likely to have an incident NTM infection than those treated without immunosuppressants. ${ }^{26}$

\section{Trend of Increasing PNTM Prevalence over Time}

Several studies have noted an increasing prevalence of PNTM over time, both of isolation and of those meeting ATS/IDSA disease criteria (- Table 1). ${ }^{1-3,13,17,27}$ Adjemian et al and Marras et al identified increases of $\sim 8 \%$ per year in independently conducted studies over a similar time period in the United States and Canada. ${ }^{1,3}$ Similarly, both HMO studies conducted across five U.S. sites observed increases over time, although at rates ranging from 2.5 to $6 \%$ per year, ${ }^{2,13}$ which is also consistent with the increases observed in the U.S. CF population. ${ }^{19}$ Du Moulin et $\mathrm{al}^{27}$ demonstrated a fivefold increase in the number of patients with MAC isolates in Massachusetts from 1972 to 1983 , suggesting that this trend of an increasing prevalence has been occurring prior to these recent studies, at least in some areas. The increase in PNTM may represent a true increase in disease, or a combination of factors including improved diagnostics and heightened awareness of NTM. For instance, improved mycobacterial diagnostics and increased use of imaging technology such as computed tomography scans ${ }^{28}$ may have resulted in more accurate detection and therefore an increased diagnosis of NTM infections among patients. Nonetheless, increases in NTM isolation and disease prevalence have been detected worldwide and over various time periods, ${ }^{2-4,13,29-34}$ suggesting that at least some of the observed increase is likely true even after considering increased awareness and diagnosis.

The increasing prevalence of PNTM has been hypothesized to be a result of increased concentration of NTM in the environment related to the passage of the Clean Water Act in 1970. This Act led to widespread disinfection of drinking water and may have inadvertently selected for increased levels of mycobacteria due to their inherent resistance to chlorination relative to other competing microorganisms. ${ }^{35}$ However, that change implemented during the 1970s may not explain the gradual and continued increase seen during the 2000s, which could be partly explained by increased awareness and diagnosis, along with rapid molecular probes for species identification. Additionally, as noted earlier, therapies that inhibit TNF- $\alpha$, a cytokine which mediates host immune defense against opportunistic pathogens, have become widely used within the last decade to treat autoimmune inflammatory conditions such as RA, thus increasing the risk of NTM infection in these patients and perhaps contributing to its increasing prevalence. ${ }^{12,24,36}$ However, increases in TNF- $\alpha$ inhibitor exposure would not account for the increased prevalence observed in the general population not receiving these medications. Another factor that could contribute to observed increases in numbers of cases over time is the aging population, as older adults are at increased risk for PNTM ${ }^{1,2,18}$ and the proportion of Americans over age 65 years is expected to nearly double by 2030. ${ }^{37}$ However, most prevalence estimates were ageadjusted, therefore limiting the impact of this in any observed increases over time. Systematic monitoring of PNTM isolation and ATS/IDSA-defined disease is needed to better understand trends over time and any factors influencing observed changes.

\section{PNTM Treatment and Mortality Estimates}

Managing PNTM disease is a lengthy, complicated, and timeconsuming process, ${ }^{38}$ and some components of recommended therapy remain controversial. Although the 2007 ATS/IDSA guidelines specify evidence-based treatment regimens for PNTM disease caused by MAC, no established regimens of proven efficacy exist for $M$. abscessus, which can cause significant morbidity and mortality; rather, the guidelines suggest combination therapy of a macrolide with amikacin plus cefoxitin or imipenem, acknowledging a lack of clinical efficacy evidence for drugs other than clarithromycin. ${ }^{10}$ Even for disease where antibiotic regimens are specified, compliance among 
both providers and patients is poor, ${ }^{39,40}$ likely due to the complex nature of treatments and frequently reported drug intolerances. $^{41-43}$ In a survey of U.S. physicians conducted during 2011 to 2012, respondents were asked to extract medical record data on the treatment of their last four patients treated for NTM disease. ${ }^{39}$ Of MAC patients, only $13 \%$ were treated with a regimen consistent with ATS/IDSA guidelines, and $16 \%$ were treated with macrolide monotherapy, which can increase the risk of macrolide resistance and is associated with poor outcomes; $56 \%$ were treated with a regimen that did not include a macrolide at all. While pulmonologists were more likely to prescribe the recommended regimen (18\%) compared with infectious disease (10\%) and family/general practice/internal medicine ( $9 \%$ ) physicians, the low levels of adherence to treatment guidelines across specialties raise concerns about physician knowledge regarding PNTM treatment. Studies on treatment compliance conducted in Europe, Japan, and Australia found similar trends. ${ }^{40}$

Because of the lack of long-term follow-up data, estimates of PNTM-associated mortality are limited. In the U.S. Medicare beneficiary study, persons over age 65 years with PNTM were $40 \%$ more likely to die than noncases, with a higher risk of death among men and among persons identified as black. ${ }^{1}$ National mortality data from the National Center for Health Statistics have also been used to estimate the NTM-associated mortality rate based on ICD codes from 1999 to $2014 .{ }^{44}$ A significant increase in non-human immunodeficiency virus (HIV)-associated mortality was observed during that period, while the proportion of HIV-associated deaths decreased from 33\% in 1999 to $4 \%$ in $2014 .{ }^{44}$ Thus, in an era where antiretrovirals are now widely used in the HIVinfected population, the burden of NTM has shifted to the HIV-negative population. Mortality was also assessed among Oregon patients with either NTM disease or NTM isolation and a 5-year age-adjusted mortality rate of 28.7 per 1,000 persons was found among those meeting ATS disease criteria (NTM disease) versus 23.4 per 1,000 persons among those not meeting ATS disease criteria (NTM isolation). ${ }^{45}$

\section{PNTM Risk Factors}

Risk of PNTM disease is determined by the interaction of environmental, microbial, and host factors. A variety of host factors favor growth of mycobacteria and increase disease risk, including structural, immunologic, and genetic differences. Structural defects associated with NTM include conditions such as COPD, which has been identified in 18 to $38 \%$ of patients with NTM. ${ }^{1,13,23,46}$ Lung cancer is also associated with NTM lung disease, with an increased prevalence identified among male Medicare beneficiaries with PNTM. ${ }^{1}$ An additional single-center study found that $25 \%$ of lung cancer patients had a positive MAC culture compared with only $3 \%$ of patients undergoing bronchoscopy for nonbronchiectatic benign lung disease. ${ }^{47}$ Disorders of mucociliary clearance, including $\mathrm{CF}^{9,48}$ and $\mathrm{PCD},{ }^{25}$ are associated with high rates of PNTM disease. Low ciliary beat frequency has also been associated with increased PNTM disease risk in a casecontrol study of patients not diagnosed with these other conditions. $^{49}$ Certain treatment for these lung disorders, specifically immunologic therapy, can confer increased risk by inhibiting the immune response to mycobacteria. TNF- $\alpha$ blockers have been repeatedly associated with NTM risk in both cohort studies and case-control studies, as have steroids and other immunosuppressive or immunomodulatory medications. ${ }^{24,46,50,51}$ Within the CF population, chronic macrolide use appears to be protective against the development of an initial PNTM infection. ${ }^{19,52}$

In addition to predisposing structural lung abnormalities, factors, family and genetic association studies in patients without known associated conditions found that PNTM disease was correlated with low body mass index, thoracic skeletal abnormalities, mitral valve prolapse, and connective tissue disorders. ${ }^{46,53,54}$ PNTM disease and these associated traits also appear to cluster in families, suggesting common genetic risk factors. ${ }^{54,55} \mathrm{~A}$ whole-exome sequencing study using a candidate gene approach found that patients had more low-frequency variants in genes related to immune function, ciliary movement, connective tissue, as well as in the gene coding for the $\mathrm{CF}$ transmembrane conductance regulator (CFTR) protein (which causes CF when both copies are nonfunctional) compared with both unaffected relatives and control subjects. ${ }^{56}$ Another study evaluating 13 genetic variants of the CFTR gene among Korean patients by wholeexome sequencing found that the $\mathrm{Q} 1352 \mathrm{H}$ mutation showed a significantly higher frequency in PNTM patients than in controls, suggesting that this CFTR gene variant may increase susceptibility to NTM lung disease. ${ }^{57}$ Similarly, Colombo et al found known CFTR genetic variants or novel mutations in $42 \%$ ( 5 of 12 ) of NTM patients evaluated with familial disease, ${ }^{55}$ while other studies reported a prevalence of CFTR mutations among PNTM cases ranging from 37 to $50 \%{ }^{53,58}$

In addition to host-specific risk factors, tremendous geographic variation in both PNTM prevalence and mycobacterial species distribution has been noted throughout the United States, highlighting the importance of environmental risk factors in the development of PNTM disease. Historically, evidence for geographic variation in NTM exposure came from a $M$. intracellulare skin test sensitization study conducted among Navy recruits who were lifelong residents of a single county, which found that reactivity to the Battey antigen, derived from $M$. intracellulare, was higher among recruits from the Southeastern and Southwestern United States. ${ }^{59}$ More recently, disease prevalence and clustering has been associated with climatic factors at the population level, with disease clustering associated with factors related to moisture in the air. Medicare claims data examined at the county level identified seven significant clusters, or high-risk areas, of PNTM cases encompassing parts of California, Florida, Hawaii, Louisiana, New York, Oklahoma, Pennsylvania, and Wisconsin. ${ }^{7}$ Compared with low-risk counties, these areas had a higher proportion of surface water and higher mean daily potential evapotranspiration, suggesting possible roles for climatic factors in PNTM disease risk. Soil factors were also identified as important, with high-risk counties having higher levels of copper and sodium, and lower levels of manganese in the soil. ${ }^{7}$ The importance of climatic factors 
was also identified in a study of persons with $\mathrm{CF}$, where the most predictive factor of PNTM disease was the saturated vapor pressure associated with his or her residential zip code. $^{9}$ This is similar to another study of patients at $21 \mathrm{CF}$ centers that correlated PNTM prevalence with the average annual atmospheric water vaper content. ${ }^{60}$

Environments that foster greater levels of moisture in the atmosphere appear to increase the risk for NTM exposure and subsequent pulmonary disease. In particular, several studies have now consistently identified Hawaii as the highest-risk state in the country for PNTM, independent of hostlevel factors such as race/ethnicity. ${ }^{1,2,7,9,19,61}$ Additionally, data from both the Hawaii HMO study ${ }^{2}$ and another study of military-affiliated persons with $\mathrm{CF}$ residing in Hawaii ${ }^{62}$ suggest that increased duration of residence in Hawaii is associated with increased risk of PNTM infection, likely due to a longer duration of sustained, high-level environmental exposure. It is possible that this increased potential for environmental exposure in Hawaii is due to unique conditions present on the islands. ${ }^{63}$ For instance, many of the zonal soil groups in the Hawaiian Islands are classified as being humic, ${ }^{64}$ and soil samples containing humic acid as a principal component have been associated with high numbers of NTM. ${ }^{65}$ These environmental associations are also consistent with previous microbiologic findings that environmental prevalence of NTM is related to warmer temperature, low dissolved oxygen, high soluble zinc, low $\mathrm{pH}$, high humic acid, and high fulminic acid. ${ }^{65}$

Environmental risk factors for NTM may vary dependent on species, which in turn have different clinical relevance. One study based on microbiologic data from a nationally distributed electronic health record database found that species prevalence varies by region, with the proportion of isolates identified as MAC ranging from $61 \%$ in the West South Central region (states included AR, LA, OK, TX) to $91 \%$ in the East South Central region (AL, KY, MS, TN). ${ }^{23}$ The proportion of $M$. abscessus/M. chelonae isolates also varied significantly from $2 \%$ in the East South Central region to $18 \%$ in the West South Central region. Another study among CF patients had similar findings, with a greater percentage of positive mycobacterial cultures detected in southern and southeastern states due to infection from $M$. abscessus compared with most western and mid-western states, where MAC was responsible for most infections observed. Furthermore, the percentage of isolates identified as MAC ranged greatly by state, from only $29 \%$ in Louisiana to $100 \%$ in Nebraska. ${ }^{9}$ These results suggest that environmental risk factors for infection may vary by species, which has important implications for patients as treatment practices and clinical outcomes are species specific.

Several studies have also suggested that a household's water source and water pipe biofilms represent a potentially important source of NTM exposure. Two studies used repetitive sequence-based PCR to identify genetic matches between variants in environmental samples from patient households and clinical isolates from these same patients. ${ }^{66,67}$ This led to an investigation of factors affecting NTM recovery from household water, and the discovery that households with water heaters set at temperatures above $55^{\circ}$ C had a lower rate of recovery of NTM. ${ }^{66}$ However, significant variability in heat susceptibility of mycobacteria has been demonstrated in a laboratory setting, leading to concerns that alterations to water heater settings might select for more thermoresistant, and potentially more pathogenic, strains such as M. xenopi. ${ }^{68}$ Another factor that appears to influence risk of PNTM is the watershed affiliated with a patient's area of residence. ${ }^{69}$ Using data on non-CF NTM patients treated at National Jewish Health and residing in Colorado, researchers found that three specific watersheds were at increased risk of PNTM, all of which appear to share a similar water source and distribution mechanism. ${ }^{69}$

Soil and dust have also been identified as potential sources of NTM exposure. Aerosols generated from potting soils in households of PNTM patients were found to contain known pathogenic NTM species, including M. avium, M. intracellulare, and $M$. kansasii, some of which matched corresponding patient isolates by pulsed-field gel electrophoresis genotyping. ${ }^{70} \mathrm{Simi}$ lar findings were obtained from a study examining soil samples from patient households in Japan, which found NTM in potting soil, residential yard soil, and farm soil. Using variable number tandem repeat genotyping, matches between isolates obtained from residential soil samples and directly from patients were identified for six patients with high soil exposure. ${ }^{71}$ One study conducted in Palm Beach County, Florida, obtained detailed measurements on the intensity of soil-related exposures and found that participation in occupations with greater amounts of soil exposure was significantly associated with a positive $M$. avium skin test reaction, with a dose-response relationship. ${ }^{72}$

Behavioral exposures for NTM have been difficult to assess due to the rarity of the disease, ubiquity of the organism, and high frequency of common potential household and environmental exposures. Case-control studies conducted in both high-risk and general populations have identified some factors associated with NTM infection or disease. A nested casecontrol study among CF patients assessed exposures that occurred within a 4-month window prior to the patient's first positive NTM culture, compared with control subjects with negative cultures throughout the study period. ${ }^{60} \mathrm{~A}$ wide range of exposures were examined, including showering, residential, and drinking water supply; soil exposure through gardening; and nebulizer use, but key factors significantly associated with incident infection included indoor swimming pool use in the previous 4 months and observing tap water appearing rusty or unclear. ${ }^{60}$ In the general population, a case-control study in Oregon found that out of a list of water aerosol-generating activities including showering and Jacuzzi use, only spraying plants with a spray-bottle was significantly positively correlated with disease. ${ }^{46}$ The same study found that dishwashing by hand and swimming in the past 5 years (indoor or outdoor) were protective against NTM; however, this is thought to be the result of a bias toward these activities in healthier people. ${ }^{46}$ None of the soil aerosol-generating activities, including potting plants, gardening, and lawn maintenance, were significantly associated with disease. ${ }^{46}$ While neither of these two U.S.-based case-control studies found a relationship between soil exposure and PNTM disease, a case-control study 
of bronchiectasis patients in Japan with and without PNTM disease found that case-patients were more likely to have high levels of soil exposure ( $\geq 2$ hours per day). ${ }^{73}$ Similarly, a Florida-based study identified a dose-response relationship between occupational soil exposure and NTM risk. ${ }^{72}$ These differing results suggest that the routes of exposure vary by setting and population.

While NTM is primarily acquired from the environment, recent episodes of $M$. abscessus transmission among CF patients have been detected. Outbreaks of M. abscessus subsp. massiliense have been identified at multiple CF centers through whole-genome sequencing or other genetic analyses of patient isolates. ${ }^{74-76}$ Whole-genome sequencing has also detected a high level of relatedness among outbreak strains from the United States and United Kingdom. ${ }^{76,77}$ The small $(<20)$ differences in single nucleotide polymorphisms between outbreak strains have been suggested as possible proof of recent transmission between continents, although geographical differences in large-scale deletions and insertions raises the possibility of independent selection of more transmissible strains in both countries with local evolution. ${ }^{76-78}$ Additionally, contact tracing has not identified transmission routes between outbreaks; however, a recent laboratory study indicated M. abscessus may be viable in fomites. ${ }^{79}$

\section{Extrapulmonary NTM Epidemiology}

Extrapulmonary manifestations of NTM disease include disseminated, skin, joint, and lymph node infections. These infections differ greatly from PNTM disease in terms of both prevalence in the United States and associated risk factors, especially regarding the host, with a different population typically affected compared with those who are at increased risk for PNTM disease. Individuals susceptible to extrapulmonary NTM infections, especially disseminated disease, typically include those with other comorbidities that result in a compromised immune system, such as underlying immunologic disorders and HIV infection. ${ }^{80,81}$ Additionally, unlike PNTM disease, extrapulmonary NTM infections can be associated with medical or cosmetic procedures that expose a wound to sources contaminated with mycobacteria. ${ }^{82}$

While population-based studies exist for PNTM, few current studies describe the epidemiology of extrapulmonary NTM in the United States. One recent study in Oregon evaluated the prevalence of extrapulmonary NTM using statewide population-based laboratory surveillance data from 2007 to $2012 .^{80}$ These researchers estimated an annual incidence of extrapulmonary NTM infection of 1.5 cases per 100,000 population, which was stable over time. Extrapulmonary NTM patients were younger on average than PNTM patients, with a median age of 51 years. Additionally, while half of the cases identified had MAC, rapid-growing NTM species were identified at a much greater frequency than among PNTM patients within the state and represented a third of all cases. ${ }^{80}$ These findings are similar to results from another recent study utilizing a large electronic medical record database, with linked laboratory, demographic, and clinical information for hospitalized patients from 156 hospitals throughout the United States between 2005 and $2015 .{ }^{81}$ There, extrapulmonary NTM incidence was estimated at 1.9 cases per 100,000 persons, $55 \%$ of which represented skin and soft-tissue infections. Common comorbidities identified among these cases included fungal infections (16\%) and HIV (14\%). Like in the Oregon study, just over half of the cases had MAC infections and 26\% had infections with rapidgrowing NTM. This study also identified regional differences in species, with $81 \%$ of all M. abscessus cases in the South and 67\% of MAC cases in the Northeast.

\section{Research Needs}

Epidemiological studies performed thus far highlight the increasing prevalence of NTM in the United States and its disproportionate burden on older populations and persons of Asian ancestry, with varying risk in different Asian subpopulations, especially in high-risk environments. ${ }^{1,2}$ To further elucidate the risk and burden of NTM disease, population-level research is needed in several disparate areas, including obtaining more detailed species-specific information on environmental reservoirs and geographic clustering of disease by species, as well as clinical studies to further identify genes associated with disease susceptibility. Regarding treatment, given that guidelines do not recommend universal treatment following NTM diagnosis due to the high cost, long duration, and significant side effects of the currently available therapies, further study of factors determining the treatment success and associated outcomes is warranted. In addition, poor treatment outcomes may be mediated in great part by antibiotic resistance, but the risk of developing antibiotic resistance has not been well studied. For species such as M. abscessus, which may have resistance to first-line antibiotics (e.g., macrolides) prior to treatment, studies of the frequency of antibiotic resistance among isolates from treatment-naïve patients would also be useful. Moreover, the high rates of reinfection seen in singlecenter studies support the importance of host susceptibility, and support the idea of studying risk factors for infection or reinfection in these most vulnerable populations. Lastly, a few recent studies identified a potential dose-response relationship with greater numbers of years residing in high-risk environments, such as Hawaii, and the risk of NTM acquisition among both the general population ${ }^{2}$ and among persons with $\mathrm{CF}^{62}$ More research is needed to understand biological mechanisms that drive the increased risk and prevalence of NTM observed with greater duration of high environmental exposure levels, especially in particularly vulnerable populations like individuals with $\mathrm{CF}$.

\section{References}

1 Adjemian J, Olivier KN, Seitz AE, Holland SM, Prevots DR. Prevalence of nontuberculous mycobacterial lung disease in U.S. Medicare beneficiaries. Am J Respir Crit Care Med 2012;185(08):881-886

2 Adjemian J, Frankland TB, Daida YG, et al. Epidemiology of nontuberculous mycobacterial lung disease and tuberculosis, Hawaii, USA. Emerg Infect Dis 2017;23(03):439-447

3 Marras TK, Chedore P, Ying AM, Jamieson F. Isolation prevalence of pulmonary non-tuberculous mycobacteria in Ontario, 19972003. Thorax 2007;62(08):661-666 
4 Thomson RM; NTM Working Group at Queensland TB Control Centre and Queensland Mycobacterial Reference Laboratory. Changing epidemiology of pulmonary nontuberculous mycobacteria infections. Emerg Infect Dis 2010;16(10):1576-1583

5 Ko RE, Moon SM, Ahn S, et al. Changing epidemiology of nontuberculous mycobacterial lung diseases in a tertiary referral hospital in Korea between 2001 and 2015. J Korean Med Sci 2018;33(08):e65

6 Shah NM, Davidson JA, Anderson LF, et al. Pulmonary Mycobacterium avium-intracellulare is the main driver of the rise in nontuberculous mycobacteria incidence in England, Wales and Northern Ireland, 2007-2012. BMC Infect Dis 2016;16:195

7 Adjemian J, Olivier KN, Seitz AE, Falkinham JO III, Holland SM, Prevots DR. Spatial clusters of nontuberculous mycobacterial lung disease in the United States. Am J Respir Crit Care Med 2012;186 (06):553-558

8 Falkinham JO III. Surrounded by mycobacteria: nontuberculous mycobacteria in the human environment. J Appl Microbiol 2009; 107(02):356-367

9 Adjemian J, Olivier KN, Prevots DR. Nontuberculous mycobacteria among patients with cystic fibrosis in the United States: screening practices and environmental risk. Am J Respir Crit Care Med 2014; 190(05):581-586

10 Griffith DE, Aksamit T, Brown-Elliott BA, et al; ATS Mycobacterial Diseases Subcommittee; American Thoracic Society; Infectious Disease Society of America. An official ATS/IDSA statement: diagnosis, treatment, and prevention of nontuberculous mycobacterial diseases. Am J Respir Crit Care Med 2007;175(04): 367-416

11 Okoro CA, Zhao G, Fox JB, Eke PI, Greenlund KJ, Town M. Surveillance for health care access and health services use, adults aged 18-64 years - Behavioral Risk Factor Surveillance System, United States, 2014. MMWR Surveill Summ 2017;66(07):1-42

12 Winthrop KL, Baxter R, Liu L, et al. The reliability of diagnostic coding and laboratory data to identify tuberculosis and nontuberculous mycobacterial disease among rheumatoid arthritis patients using anti-tumor necrosis factor therapy. Pharmacoepidemiol Drug Saf 2011;20(03):229-235

13 Prevots DR, Shaw PA, Strickland D, et al. Nontuberculous mycobacterial lung disease prevalence at four integrated health care delivery systems. Am J Respir Crit Care Med 2010;182(07):970-976

14 Smith GS, Ghio AJ, Stout JE, et al. Epidemiology of nontuberculous mycobacteria isolations among central North Carolina residents, 2006-2010. J Infect 2016;72(06):678-686

15 Winthrop KL, McNelley E, Kendall B, et al. Pulmonary nontuberculous mycobacterial disease prevalence and clinical features: an emerging public health disease. Am J Respir Crit Care Med 2010; 182(07):977-982

16 Henkle E, Hedberg K, Schafer S, Novosad S, Winthrop KL. Population-based incidence of pulmonary nontuberculous mycobacterial disease in Oregon 2007 to 2012. Ann Am Thorac Soc 2015;12 (05):642-647

17 Donohue MJ, Wymer L. Increasing prevalence rate of nontuberculous mycobacteria infections in five states, 2008-2013. Ann Am Thorac Soc 2016;13(12):2143-2150

18 Strollo SE, Adjemian J, Adjemian MK, Prevots DR. The burden of pulmonary nontuberculous mycobacterial disease in the United States. Ann Am Thorac Soc 2015;12(10):1458-1464

19 Adjemian J, Olivier KN, Prevots DR. Epidemiology of pulmonary nontuberculous mycobacterial sputum positivity in patients with cystic fibrosis in the United States, 2010-2014. Ann Am Thorac Soc 2018;15(07):817-826

20 Smith MJ, Efthimiou J, Hodson ME, Batten JC. Mycobacterial isolations in young adults with cystic fibrosis. Thorax 1984;39 (05):369-375

21 Knapp EA, Fink AK, Goss CH, et al. The Cystic Fibrosis Foundation Patient Registry. Design and methods of a national observational disease registry. Ann Am Thorac Soc 2016;13(07):1173-1179
22 McKone EF, Goss CH, Aitken ML. CFTR genotype as a predictor of prognosis in cystic fibrosis. Chest 2006;130(05):1441-1447

23 Spaulding AB, Lai YL, Zelazny AM, et al. Geographic distribution of nontuberculous mycobacterial species identified among clinical isolates in the United States, 2009-2013. Ann Am Thorac Soc 2017;14(11):1655-1661

24 Brode SK, Jamieson FB, Ng R, et al. Increased risk of mycobacterial infections associated with anti-rheumatic medications. Thorax 2015;70(07):677-682

25 Noone PG, Leigh MW, Sannuti A, et al. Primary ciliary dyskinesia: diagnostic and phenotypic features. Am J Respir Crit Care Med 2004;169(04):459-467

26 Chao WC, Lin $\mathrm{CH}$, Liao TL, et al. The risk of nontuberculous mycobacterial infection in patients with Sjögren's syndrome: a nationwide, population-based cohort study. BMC Infect Dis 2017; 17(01):796

27 du Moulin GC, Sherman IH, Hoaglin DC, Stottmeier KD. Mycobacterium avium complex, an emerging pathogen in Massachusetts. J Clin Microbiol 1985;22(01):9-12

28 Seitz AE, Olivier KN, Adjemian J, Holland SM, Prevots DR. Trends in bronchiectasis among Medicare beneficiaries in the United States, 2000 to 2007. Chest 2012;142(02):432-439

29 Sakatani M. [The epidemiology of pulmonary disease caused by Mycobacterium avium complex in Japan]. Kekkaku 1993;68(01): 43-46

30 O'Brien DP, Currie BJ, Krause VL. Nontuberculous mycobacterial disease in northern Australia: a case series and review of the literature. Clin Infect Dis 2000;31(04):958-967

31 Pang SC. Mycobacterium kansasii infections in Western Australia (1962-1987). Respir Med 1991;85(03):213-218

32 Wang HX, Yue J, Han M, et al. Nontuberculous mycobacteria: susceptibility pattern and prevalence rate in Shanghai from 2005 to 2008. Chin Med J (Engl) 2010;123(02):184-187

33 Ding LW, Lai CC, Lee LN, Hsueh PR. Disease caused by nontuberculous mycobacteria in a university hospital in Taiwan, 1997-2003. Epidemiol Infect 2006;134(05):1060-1067

34 Park YS, Lee CH, Lee SM, et al. Rapid increase of non-tuberculous mycobacterial lung diseases at a tertiary referral hospital in South Korea. Int J Tuberc Lung Dis 2010;14(08):1069-1071

35 Falkinham JO. Impact of human activities on the ecology of nontuberculous mycobacteria. Future Microbiol 2010;5(06):951-960

36 Winthrop KL, Chang E, Yamashita S, Iademarco MF, LoBue PA Nontuberculous mycobacteria infections and anti-tumor necrosis factor-alpha therapy. Emerg Infect Dis 2009;15(10):1556-1561

37 Colello K. Where Do Older Americans Live? Geographic Distribution of the Older Population. Washington, DC: Division of Digital Signal Processing; 2007

38 Griffith DE, Winthrop KL. Mycobacterium avium complex lung disease therapy. Am J Respir Crit Care Med 2012;186(06):477-479

39 Adjemian J, Prevots DR, Gallagher J, Heap K, Gupta R, Griffith D. Lack of adherence to evidence-based treatment guidelines for nontuberculous mycobacterial lung disease. Ann Am Thorac Soc 2014;11(01):9-16

40 van Ingen J, Wagner D, Gallagher J, et al; NTM-NET. Poor adherence to management guidelines in nontuberculous mycobacterial pulmonary diseases. Eur Respir J 2017;49(02):49

41 van Ingen J, Egelund EF, Levin A, et al. The pharmacokinetics and pharmacodynamics of pulmonary Mycobacterium avium complex disease treatment. Am J Respir Crit Care Med 2012;186(06):559-565

42 Field SK, Fisher D, Cowie RL. Mycobacterium avium complex pulmonary disease in patients without HIV infection. Chest 2004;126(02):566-581

43 Huang JH, Kao PN, Adi V, Ruoss SJ. Mycobacterium avium-intracellulare pulmonary infection in HIV-negative patients without preexisting lung disease: diagnostic and management limitations. Chest 1999;115(04):1033-1040

44 Vinnard C, Longworth S, Mezochow A, Patrawalla A, Kreiswirth BN, Hamilton K. Deaths related to nontuberculous mycobacterial 
infections in the United States, 1999-2014. Ann Am Thorac Soc 2016;13(11):1951-1955

45 Novosad SA, Henkle E, Schafer S, et al. Mortality after respiratory isolation of nontuberculous mycobacteria: a comparison of patients who did and did not meet disease criteria. Ann Am Thorac Soc 2017;14(07):1112-1119

46 Dirac MA, Horan KL, Doody DR, et al. Environment or host?: A casecontrol study of risk factors for Mycobacterium avium complex lung disease Am J Respir Crit Care Med 2012;186(07):684-691

47 Lande L, Peterson DD, Gogoi R, et al. Association between pulmonary Mycobacterium avium complex infection and lung cancer. J Thorac Oncol 2012;7(09):1345-1351

48 Olivier KN, Weber DJ, Wallace RJ Jr, et al; Nontuberculous Mycobacteria in Cystic Fibrosis Study Group. Nontuberculous mycobacteria. I: multicenter prevalence study in cystic fibrosis. Am J Respir Crit Care Med 2003;167(06):828-834

49 Fowler CJ, Olivier KN, Leung JM, et al. Abnormal nasal nitric oxide production, ciliary beat frequency, and Toll-like receptor response in pulmonary nontuberculous mycobacterial disease epithelium. Am J Respir Crit Care Med 2013;187(12):1374-1381

50 Winthrop KL, Baxter R, Liu L, et al. Mycobacterial diseases and antitumour necrosis factor therapy in USA. Ann Rheum Dis 2013; 72(01):37-42

51 Brode SK, Jamieson FB, Ng R, et al. Risk of mycobacterial infections associated with rheumatoid arthritis in Ontario, Canada. Chest 2014;146(03):563-572

52 Binder AM, Adjemian J, Olivier KN, Prevots DR. Epidemiology of nontuberculous mycobacterial infections and associated chronic macrolide use among persons with cystic fibrosis. Am J Respir Crit Care Med 2013;188(07):807-812

53 Kim RD, Greenberg DE, Ehrmantraut ME, et al. Pulmonary nontuberculous mycobacterial disease: prospective study of a distinct preexisting syndrome. Am J Respir Crit Care Med 2008;178 (10):1066-1074

54 Leung JM, Fowler C, Smith C, et al. A familial syndrome of pulmonary nontuberculous mycobacteria infections. Am J Respir Crit Care Med 2013;188(11):1373-1376

55 Colombo RE, Hill SC, Claypool RJ, Holland SM, Olivier KN. Familial clustering of pulmonary nontuberculous mycobacterial disease. Chest 2010;137(03):629-634

56 Szymanski EP, Leung JM, Fowler CJ, et al. Pulmonary nontuberculous mycobacterial infection. A multisystem, multigenic disease. Am J Respir Crit Care Med 2015;192(05):618-628

57 Jang MA, Kim SY, Jeong BH, et al. Association of CFTR gene variants with nontuberculous mycobacterial lung disease in a Korean population with a low prevalence of cystic fibrosis. J Hum Genet 2013;58(05):298-303

58 Ziedalski TM, Kao PN, Henig NR, Jacobs SS, Ruoss SJ. Prospective analysis of cystic fibrosis transmembrane regulator mutations in adults with bronchiectasis or pulmonary nontuberculous mycobacterial infection. Chest 2006;130(04):995-1002

59 Edwards LB, Acquaviva FA, Livesay VT, Cross FW, Palmer CE. Clinical and laboratory studies of tuberculosis and respiratory disease: the navy recruit program. Am Rev Respir Dis 1969;99:1-132

60 Prevots DR, Adjemian J, Fernandez AG, Knowles MR, Olivier KN. Environmental risks for nontuberculous mycobacteria. Individual exposures and climatic factors in the cystic fibrosis population. Ann Am Thorac Soc 2014;11(07):1032-1038

61 Mirsaeidi M, Machado RF, Garcia JG, Schraufnagel DE. Nontuberculous mycobacterial disease mortality in the United States, 1999-2010: a population-based comparative study. PLoS One 2014;9(03):e91879

62 Adjemian J, Prevots DR, Ricotta E, et al. Epidemiology of nontuberculous mycobacterial lung disease among cystic fibrosis patients residing in Hawaii, USA. IDWeek; San Diego, CA; 2017

63 Hennessee CT, Seo JS, Alvarez AM, Li QX. Polycyclic aromatic hydrocarbon-degrading species isolated from Hawaiian soils: Mycobacterium crocinum sp. nov., Mycobacterium pallens sp. nov., Mycobacterium rutilum sp. nov., Mycobacterium rufum sp. nov. and Mycobacterium aromaticivorans sp. nov. Int J Syst Evol Microbiol 2009;59(Pt 2):378-387

64 United States Department of Agriculture. Soil survey of the territory of Hawaii: Islands of Hawaii, Kauaii, Lanai, Maui, Molokai, and Oahu. Soil Survey Series 1955;1939:1-64

65 Kirschner RA Jr, Parker BC, Falkinham JO III. Epidemiology of infection by nontuberculous mycobacteria. Mycobacterium avium, Mycobacterium intracellulare, and Mycobacterium scrofulaceum in acid, brown-water swamps of the southeastern United States and their association with environmental variables. Am Rev Respir Dis 1992;145(2 Pt 1):271-275

66 Falkinham JO III. Nontuberculous mycobacteria from household plumbing of patients with nontuberculous mycobacteria disease. Emerg Infect Dis 2011;17(03):419-424

67 Thomson R, Tolson C, Carter R, Coulter C, Huygens F, Hargreaves $M$. Isolation of nontuberculous mycobacteria (NTM) from household water and shower aerosols in patients with pulmonary disease caused by NTM. J Clin Microbiol 2013;51(09):3006-3011

68 Schulze-Röbbecke R, Buchholtz K. Heat susceptibility of aquatic mycobacteria. Appl Environ Microbiol 1992;58(06):1869-1873

69 Lipner EM, Knox D, French J, Rudman J, Strong M, Crooks JL. A geospatial epidemiologic analysis of nontuberculous mycobacterial infection: an ecological study in Colorado. Ann Am Thorac Soc 2017;14(10):1523-1532

70 De Groote MA, Pace NR, Fulton K, Falkinham JO III. Relationships between Mycobacterium isolates from patients with pulmonary mycobacterial infection and potting soils. Appl Environ Microbiol 2006;72(12):7602-7606

71 Fujita K, Ito Y, Hirai T, et al. Genetic relatedness of Mycobacterium avium-intracellulare complex isolates from patients with pulmonary MAC disease and their residential soils. Clin Microbiol Infect 2013;19(06):537-541

72 Reed C, von Reyn CF, Chamblee S, et al. Environmental risk factors for infection with Mycobacterium avium complex. Am J Epidemiol 2006;164(01):32-40

73 Maekawa K, Ito Y, Hirai T, et al. Environmental risk factors for pulmonary Mycobacterium avium-intracellulare complex disease. Chest 2011;140(03):723-729

74 Aitken ML, Limaye A, Pottinger P, et al. Respiratory outbreak of Mycobacterium abscessus subspecies massiliense in a lung transplant and cystic fibrosis center. Am J Respir Crit Care Med 2012; 185(02):231-232

75 Bryant JM, Grogono DM, Greaves D, et al. Whole-genome sequencing to identify transmission of Mycobacterium abscessus between patients with cystic fibrosis: a retrospective cohort study. Lancet 2013;381(9877):1551-1560

76 Bryant JM, Grogono DM, Rodriguez-Rincon D, et al. Emergence and spread of a human-transmissible multidrug-resistant nontuberculous mycobacterium. Science 2016;354(6313):751-757

77 Tettelin H, Davidson RM, Agrawal S, et al. High-level relatedness among Mycobacterium abscessus subsp. massiliense strains from widely separated outbreaks. Emerg Infect Dis 2014;20(03):364-371

78 Strong M, Davidson RM. Microbiology: bacterial transmission tactics. Nature 2017;543(7646):495-496

79 Malcolm KC, Caceres SM, Honda JR, et al. Mycobacterium abscessus displays fitness for fomite transmission. Appl Environ Microbiol 2017;83(19):83

80 Henkle E, Hedberg K, Schafer SD, Winthrop KL. Surveillance of extrapulmonary nontuberculous mycobacteria infections, Oregon, USA, 2007-2012. Emerg Infect Dis 2017;23(10):1627-1630

81 Adjemian J, Lai Y, Ricotta E. DR P. Epidemiology of extrapulmonary nontuberculous mycobacterial infections among hospitalized patients in the United States American Thoracic Society Annual Conference; 2018; San Diego, CA

82 Piersimoni C, Scarparo C. Extrapulmonary infections associated with nontuberculous mycobacteria in immunocompetent persons. Emerg Infect Dis 2009;15(09):1351-1358, quiz 1544 